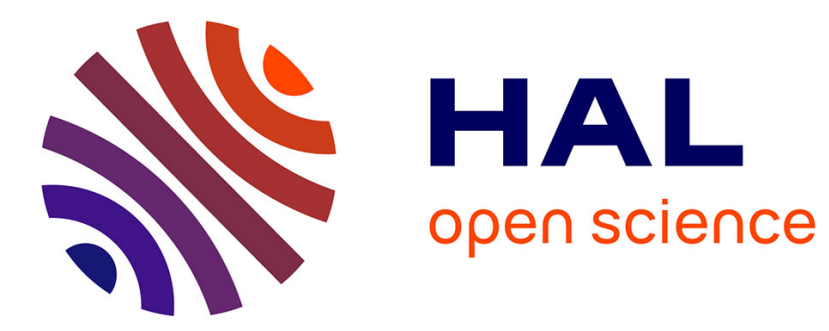

\title{
Les Téphras du Pariou (Chaine des Puys, France) : nouvelles observations sur la dispersion proximale, apports téphrostratigraphiques
}

Didier Miallier, Pierre Boivin, Thierry Pilleyre, L. Choupin, D. Malterre, S. Sanzelle

\section{To cite this version:}

Didier Miallier, Pierre Boivin, Thierry Pilleyre, L. Choupin, D. Malterre, et al.. Les Téphras du Pariou (Chaine des Puys, France) : nouvelles observations sur la dispersion proximale, apports téphrostratigraphiques. Quaternaire, 2008, 19, pp.87-96. in2p3-00297500

\section{HAL Id: in2p3-00297500 https://hal.in2p3.fr/in2p3-00297500}

Submitted on 16 Jul 2008

HAL is a multi-disciplinary open access archive for the deposit and dissemination of scientific research documents, whether they are published or not. The documents may come from teaching and research institutions in France or abroad, or from public or private research centers.
L'archive ouverte pluridisciplinaire HAL, est destinée au dépôt et à la diffusion de documents scientifiques de niveau recherche, publiés ou non, émanant des établissements d'enseignement et de recherche français ou étrangers, des laboratoires publics ou privés. 


\section{LES TEPHRAS DU PARIOU (CHAîne DES PUYS, FRANCE): NOUVELLES}

OBSERVATIONS SUR LA DISPERSION PROXIMALE ; APPORTS TEPHROSTRATIGRAPHIQUES.

D. Miallier ${ }^{(1)}$, P. Boivin ${ }^{(2)}$, T. Pilleyre ${ }^{(1)}$, L. Choupin $^{(2)}$, D. Malterre ${ }^{(2)}$ et S. Sanzelle ${ }^{(1)}$.

(1) Laboratoire de Physique Corpusculaire, UMR 6533, IN2P3 - CNRS, Université Blaise Pascal, 63177, Aubière cedex.

(2) Laboratoire Magmas et Volcans, UMR 6524 CNRS-Université Blaise Pascal, 5, rue Kessler, 63000, Clermont-Ferrand

\section{Résumé}

Le puy de Pariou est un volcan âgé de 9500 ans environ, caractérisé par une évolution concomitante de son magma et de son dynamisme. Durant la première phase, des éruptions à forte composante phréatomagmatique construisent un anneau de tufs à faciès de débourrage dont le magma juvénile, d'abord trachytique, évolue en trachy-andésite avec émission de coulées de lave. La seconde phase est celle de l'édification d'un cône strombolien qui se superpose à l'anneau de tufs, avec un magma de trachy-andésite basaltique.

De nouvelles observations mettent en évidence une anisotropie dans la dispersion des téphras à l'échelle de quelques kilomètres : ceux qui correspondent à l'anneau de tufs initial sont beaucoup plus développés à l'ouest et au sud-ouest du volcan que dans les autres directions. A l'opposé, les derniers produits liés à l'édification du cône, sont rares et n'ont été observés que vers l'est et le sud-est.

La formation occidentale, dont la puissance atteint localement 6 mètres, est constituée d'une succession de couches décimétriques de lapilli à faciès de débourrage représentant une alternance de phases phréatomagmatiques ou magmatiques. Elles débutent par un lit pluridécimétrique de ponces claires, de nature trachytique. L'épaisseur de la formation, qui s'est en partie mise en place sous forme de déferlantes, varie fortement en fonction du relief, suivant des effets de congère et de canalisation.

L'identification facile de la séquence en fait un bon repère stratigraphique, des exemples inédits en sont donnés : les tufs phréatomagmatiques du cratère du Nid de la Poule se situent sur cette formation et le sommet du puy de Côme est dessous ; une large faille de 1,6 m de largeur, orientée sensiblement N-S, s'est ouverte dans une ancienne coulée de lave au sud du puy Filhu après le dépôt des téphras du Pariou, ce qui suggère l'occurrence récente d'un événement tectonique. 
Mots -clés : Puy Pariou, téphrostratigraphie, phréatomagmatisme, faille.

\begin{abstract}
The Puy Pariou, a volcano aged c. 9,500 y, is characterised by a simultaneous evolution of both its magma and dynamism in the course of the eruptions. During the first phase, explosions that were marked by a significant phreatomagmatic contribution, have built a tuff ring, the magma of which evolved from a trachyte composition to a trachyandesite one. The second phase corresponded to the construction of a strombolian cone, with basaltic trachyandesite lava, the "Nouveau Pariou".

New field observations on the Pariou tephras have evidenced anisotropy of their spatial distribution. The tephras connected with the first phase show a south and western tropism, whereas, at the opposite, the tephras from the strombolian phase are very scarce and were observed only eastwards of the volcano.

The western formation, which is locally thicker than $6 \mathrm{~m}$, is constituted of many lapilli layers, 5 to $40 \mathrm{~cm}$ thick. Most of those layers have a mixed composition of fresh lava, old lava and lithics, which exhibit magmatic or phreatomagmatic features. The basis of the formation is a remarkable bed of light coloured pumice lapilli, the thickness of which is between 10 and 40 $\mathrm{cm}$.
\end{abstract}

Those tephras are not evenly distributed. On the contrary, their amount appears to be strongly dependent on the relief, thus indicating a prominent role of surges in the process of deposition.

Since identification of the Pariou tephras is easy, they can be considered as a useful stratigraphic marker in the centre of the Chaîne des Puys. New examples are given: (i) the Nid de la Poule, a basalt phreatomagmatic crater, which had not yet been dated, is younger than the Pariou; (ii) the puy de Côme, a trachyandesite cone for which a TL age of c 7600 y has previously been published is older than the Pariou and (iii), at 3,000 m SW of the Pariou, a $1.60 \mathrm{~m}$ wide fault opened through an old unidentified lava flow, after the Pariou eruption, thus suggesting the occurrence of a recent tectonic event.

Key words: Puy Pariou; trachyte, trachyandesite, tephrostratigraphy, fault. 


\section{1 - Introduction}

Le puy de Pariou, est l'un des volcans majeurs de la Chaîne des Puys par la taille de son cône principal (environ $200 \mathrm{~m}$ de hauteur pour $900 \mathrm{~m}$ de diamètre), la longueur des coulées qui en sont issues (environ $7 \mathrm{~km}$ pour la plus longue), et par l'étendue de la nappe de pyroclastites dont il a recouvert plusieurs volcans voisins et les marges de la plaine de Limagne (Boivin et al, 2004). Son histoire est marquée par une évolution concomitante de son dynamisme et de la nature de son magma. Durant la première phase éruptive, un dynamisme à forte composante phréatomagmatique construit un anneau de tufs (Fig. 1) dont le magma juvénile, d'abord trachytique $\left(60 \%<\mathrm{SiO}_{2}<62 \%\right)$, évolue en trachy-andésite $\left(55 \%<\mathrm{SiO}_{2}<57 \%\right)$ avec émission de coulées de lave en fin de cycle (NB. la nomenclature française des laves sera utilisée dans cet article). Cet anneau n'a pas de dénomination particulière, le terme « Ancien Pariou » étant réservé, depuis 1982 (Camus et al., 1982), à un petit cône basaltique plus ancien, sans rapport direct avec l'ensemble étudié ici et qui a presqu'entièrement été détruit par les éruptions ultérieures. La seconde phase est celle de l'édification d'un cône strombolien, le Nouveau Pariou, qui recoupe l'anneau de tufs, avec un magma de trachyandésite basique (51\%< $\mathrm{SiO}_{2}<54 \%$ ) (Camus, 1975 ; El Arabi, 1987 ; Boivin et al., 2004). Il ne semble pas y avoir eu de longue pause entre le début et la fin de tout le cycle éruptif. Sa datation est imprécise : la stratigraphie permet seulement d'affirmer que le puy Chopine $(9,72$ $\pm 0,27 \mathrm{ka}$, à $2 \sigma)$ est plus ancien et que le cratère Kilian $(9,43 \pm 0,11 \mathrm{ka})$ est plus récent (Boivin et al., 2004 ; Miallier et al., 2004). Par commodité, un âge de 9500 ans environ sera évoqué.

Depuis la dernière édition de la Carte volcanologique de la Chaîne des Puys (Boivin et al., 2004), une prospection systématique a montré que la nappe de pyroclastites attribuables au Pariou s'étend vers l'ouest bien au delà des contours précédemment retenus (Fig.1), avec de plus, une séquence téphrique plus développée que ce qui en était connu.

L'objectif de cet article est de décrire cette formation, d'en donner une interprétation dans le contexte actuel des connaissances sur le puy de Pariou et de montrer, avec des exemples inédits, que son identification facile en fait un utile repère téphrostratigraphique.

\section{2- La séquence type}

La séquence la plus développée est visible dans le front d'une petite carrière (site $\mathrm{S} 4$, Tab. 1 et Fig. 2) ouverte dans le col entre le puy Filhu et le puy Balmet. 
La base de la formation est un lit d'une vingtaine de centimètres d'épaisseur (P, Fig. 2), dont la couleur claire (gris-jaune) contraste avec les autres couches (Fig. 4). Il est constitué à 35\% (en masse) de ponces vraies $(\mathrm{d}<1)$, fortement vésiculées, de dimensions variables jusqu'à 15 $20 \mathrm{~cm}$. Les plus grosses ont parfois le cœur brun-rouge. Elles présentent fréquemment des « rubannements ». Quelques rares phénocristaux d'amphibole sont observables à l'œil nu.

A l'observation en lame mince, ces ponces ont une texture classique avec au moins deux générations de bulles. Les plus grosses, millimétriques, sont régulièrement réparties. Les plus petites, de quelques dizaines de micromètres, se forment par nucléation autour des discontinuités déjà présentes dans la lave: phénocristaux et xénolites. Certaines ponces montrent des bulles très étirées. Les échantillons montrent tous des traces de mélange entre une lave caractérisée par un verre incolore assez riche en microlites d'amphibole brun-vert, de feldspath plagioclase et d'opaques et une lave avec un verre brun plus pauvre en microlites. Les phénocristaux fréquents, en amas millimétriques, automorphes et limpides, sont constitués de plagioclase, d'amphibole brune et de titanomagnétite

Le lit de ponces, $P$, contient aussi des lapilli anguleux gris ou noirs, de densité variable, depuis une texture ponceuse jusqu'à celle d'une lave trempée. Ces lapilli sont de la même nature pétrographique et géochimique que les ponces, sous une forme moins expansée (environ $26 \%$ ). Ils peuvent aussi présenter des rubannements et contiennent quelques amphiboles. Deux analyses élémentaires (Miallier et al., 2004) ont donné une composition de trachyte pour un grain anguleux noir, dense et pour une ponce (Fig. 3). Ce niveau recèle aussi des fragments de socle (30\%), des minéraux libres et des scories noires ou rouges.

Les analyses élémentaires sont très proches de celles obtenus pour des produits semblables, échantillonnés par El Arabi (1987) à la base orientale de l'anneau de tufs du Pariou. Cet auteur, de même que Camus (1975), y décrit des «trachytes noirs présentant parfois des rubannements », associés à quelques ponces. Il relève une légère différence de composition chimique entre la partie claire et la partie sombre d'un trachyte rubanné. Il existe donc une forte parenté entre la couche $\mathrm{P}$ de la carrière $\mathrm{S} 4$ et l'anneau de tufs du Pariou.

Par endroits, sur quelques mètres de longueur, le lit de grosses ponces repose sur une couche centimétrique de même nature, mais de granulométrie beaucoup plus fine (cendres millimétriques) et plus riche en ponces ( $89 \%$ ). La composition de ces deux lits de ponces, par 
la présence de xénolithes et de lapilli trempés, est caractéristique d'un dynamisme phréatomagmatique (Wohletz et al., 1985).

Au dessus du lit $\mathrm{P}$ se développe, sans solution de continuité, en nombre variable suivant les sites, une série de couches pluri-centimétriques à pluri-décimétriques qui ont toutes, qualitativement, la même composition que $\mathrm{P}$ (ensemble $\mathrm{Lr}_{0}$, sur la Fig. 2). Elles sont cependant moins individualisées, ce qui permet de conserver, par commodité, une dénomination différente pour P. Elles sont formées d'un mélange de ponces et de lapilli gris ou noirs anguleux, de scories noires ou rouges, de socle et de minéraux libres dont les proportions et la granulométrie variables caractérisent les différentes couches. La première, par exemple, contient beaucoup de lapilli noirs, denses, centimétriques, ce qui, par contraste avec $P$, constitue un repère caractéristique sur le terrain (Fig. 4). Certains niveaux intermédiaires sont dominés par le socle. Vers le haut de la séquence différents lits s'enrichissent, jusqu'à près de $100 \%$, en scories millimétriques noires à composition de trachy-andésite identique à celle du Nouveau Pariou (C 480, Tab. 2 et Fig. 3). A quelques exceptions près (lits biseautés), les épaisseurs sont constantes.

L'analyse des courbes de «pourcentage poids vs Phi », tracées pour différentes couches de lapilli (Malterre, 2005), apporte des informations complémentaires aux précédentes sur le mode de mise en place. Leur asymétrie fréquente, traduisant un fractionnement marqué des lapilli (Fischer et Schmincke, 1984), suggère la prévalence d'un mécanisme phréatomagmatique, ce qui semble confirmé par la morphologie de grains examinés au microscope électronique. Cependant, des couches interstratifiées montrent un caractère plutôt magmatique. Les diagrammes «médiane vs indice de tri » (Wohletz et al., 1995) situent les échantillons dans la zone intermédiaire entre les retombées et les déferlantes, tantôt plus près du premier pôle (la majorité des lits, dont $\mathrm{P}$, le lit de grosses ponces), tantôt plus près du second (lit initial, de cendres ponceuses).

\section{3 - Situation et interprétation de la séquence par rapport au puy de Pariou}

La formation décrite ici a été identifiée dans un secteur ouest, sud-ouest et sud à partir du Pariou (Fig. 1 et Fig. 2). Cependant, le lit de ponces de base, P, dont l'épaisseur diminue régulièrement avec la distance, n'a été repéré qu'à l'ouest du Pariou (Fig. 1).

L'épaisseur totale de la formation est très variable et dépend du relief et de la distance au Pariou. A $1000 \mathrm{~m}$ au sud, sur le Traversin, elle pourrait atteindre des épaisseurs très importantes - plus de $20 \mathrm{~m}$ ? - (Camus, 1975) mais cela reste une hypothèse basée sur la 
topographie, sans observation en coupe. Sur le petit Suchet (S2) et sur le Cliersou (S1), à 8$900 \mathrm{~m}$ du Pariou, elle ne dépasse pas le mètre et, au col entre le puy Filhu et le puy Balmet (à $2400 \mathrm{~m}$ du Pariou, S16) seul le lit de ponces existe. Elle peut être très sporadique, presque inexistante sur certains volcans : Grand Suchet, Filhu, Balmet et Côme. Par contre, localement derrière le sommet du Côme ( $\mathrm{S} 8$, à environ $2400 \mathrm{~m}$ du Pariou) ou derrière le col entre le Filhu et le Balmet ( $\mathrm{S} 4$, à environ $2500 \mathrm{~m}$ ) les lapilli se sont accumulés par un effet de congère $(6 \mathrm{~m}$ en S4). Rapprochées de l'observation de figures de « dunes et d'anti-dunes » sur le Traversin (Camus, 1975, Boivin et al., 2004), ces remarques évoquent le rôle important d'un souffle sub-horizontal dans la mise en place des dépôts. Cette hypothèse est en accord avec le fait que la formation est très peu représentée sur les hauteurs, paraissant contrôlée en partie par le relief (canalisation entre Côme et Balmet).

La séquence présente son complet développement à l'ouest et au sud-ouest du Pariou. Au nord-est, à l'est, au sud-est du volcan et jusqu'en Limagne, seul le sommet de la séquence a été observé (Vernet et al., 2000, Choupin, 2005). Il est caractérisé notamment par une augmentation de la proportion des lapilli de trachy-andésite juvénile. La transition entre les lapilli liés à la phase de construction de l'anneau initial - supposé trachytique, jusqu'à présent - et ceux de la phase strombolienne du grand cône terminal n'apparaît pas clairement dans les coupes. En effet, si la chimie des lapilli juvéniles observés en S1 (C480) ou S18 (C523) les rattache au cône par comparaison aux analyses de El Arabi (1987), leur interstratification dans des couches de lapilli à faciès de débourrage, riches en ponces ou en éléments du socle les situe plutôt dans la dynamique de l'anneau. Pour résoudre cette contradiction, il faut supposer que la dynamique de débourrage a accompagné les premières émissions de trachy-andésite basique sans que les indices en aient été vus jusqu'à présent sur l'édifice lui-même.

Peu d'affleurements montrent des produits typiques du cône : par exemple, à la Pépinière de Royat ( $\mathrm{S} 19,6,5 \mathrm{~km}$ au sud-est du volcan), $60 \mathrm{~cm}$ de lapilli centimétriques scoriacés, à faciès de saupoudrage et à composition de trachy-andésite basique (C 344-1, Tab. 2), reposent sur la formation de produits de débourrage (C 344-5).

La composition chimique de l'ensemble des échantillons analysés pour le Pariou (Fig. 3) est marquée par un hiatus que El Arabi avait déjà noté et tenté d'interpréter : s'il n'y a pas un biais d'échantillonnage, cette absence de produits de composition intermédiaire traduit le fait que les magmas se sont très peu mélangés. Le schéma actuellement retenu est celui d'une chambre nettement stratifiée d'où auraient été expulsés successivement le magma trachytique 
qui a formé l'anneau puis le trachy-andésite à l'origine de la coulée et, enfin, le trachyandésite basique qui a construit le cône (Boivin et al., 2004).

\section{4 - Téphras sous-jacents}

\section{4-1 Inventaire}

Sur le petit Suchet et sur le Cliersou (sites S1 et S2), les ponces $\mathrm{P}$ reposent sur une couche, d'un demi mètre d'épaisseur, de lapilli émoussés à faciès de débourrage, composés de granules de lave grise, non vésiculée, mêlée d'un peu de socle (Fig. $2: \operatorname{Lr}_{2}$ et $\operatorname{Lr}_{1}$ ), ces deux couches reposant directement sur les trachytes respectifs des deux volcans (Tc et Tps). Sur le Cliersou, le trachyte est pédogénisé sur $5 \mathrm{~cm}$ d'épaisseur environ.

Vers le sommet du puy de Côme, en contrebas ouest du cratère externe (S8), à $1155 \mathrm{~m}$ d'altitude, la formation du Pariou (sans la couche P) s'étend en un placage de 40 m environ de longueur sur plus de $1 \mathrm{~m}$ d'épaisseur reposant sur des scories rouges, grossières, plus ou moins altérées en surface, probablement autochtones.

Sur une demi-circonférence du Balmet (versant est-sud-ouest) se rencontrent à peu près partout les mêmes éléments de téphrostratigraphie : à la base du Côme (S3, Fig. 2), les ponces reposent sur une couche décimétrique de lapilli squelettiques noirs ou rouille à faciès de saupoudrage $\left(\mathrm{Ls}_{0}\right)$, dont la composition est celle d'un trachy-basalte (C443, Tab. 2). Ces produits couvrent eux-mêmes des lapilli scoriacés noirs (Ls1, épaisseur supérieure à 1,5 m). Au col Filhu-Balmet (S16, Fig. 4), les ponces reposent sur une épaisse formation (plusieurs mètres) de lapilli scoriacés noirs, faiblement lités, de même aspect que $\mathrm{Ls}_{1}$. Cette formation recouvre elle-même $40 \mathrm{~cm}$ de blocs et lapilli à faciès de débourrage très riches en éléments du socle, déposés sur la surface d'une coulée de lave. Dans la carrière $\mathrm{S} 4$, des lapilli scoriacés noirs, $\mathrm{Lr}_{3}-\mathrm{Ls}_{2}$, présentant une stratification plus ou moins entrecroisée encadrent la couche $\mathrm{Ls}_{0}$ (Fig. 2). Ces lapilli sont d'un aspect très proche de $\mathrm{Ls}_{1}$ avec, pour certains lits, un léger émoussé des granules. Cette interstratification peut résulter : du fonctionnement synchrone de deux volcans ou bien de plusieurs épisodes éruptifs distincts d'un seul volcan, ou, enfin, d'un glissement gravitaire, sur une faible distance, de la formation $\mathrm{Ls}_{1}$ visible au pied du Côme et au col Filhu-Balmet. Un tel glissement, commencé avant le dépôt de $\mathrm{Ls}_{0}$ se serait poursuivi après, alimentant la couche $\mathrm{Lr}_{3}$. La même stratigraphie peut être observée depuis cette carrière (S4) jusqu'à la base occidentale du Balmet, avec une diminution régulière de sa puissance vers 1'ouest (S5, S6, Fig. 2 et Fig. 4). La partie supérieure de $\mathrm{Lr}_{3}$ est, localement, indurée dans le premier décimètre et présente alors un aspect altéré en lits centimétriques bruns ( 55 et S6). 


\section{4-2 Recherche des sources}

Il ne peut être exclu a priori que les téphras à faciès de débourrage les plus proches du Pariou, $\mathrm{Lr}_{1}$ et $\mathrm{Lr}_{2}$, soient les témoins d'une phase éruptive ancienne de ce volcan, mais la question est ouverte.

Les lapilli noirs $\mathrm{Ls}_{1}, \mathrm{Ls}_{2}$ et $\mathrm{Lr}_{3}$ ont une composition à la limite des trachy-andésites et des trachy-basaltes (C464, C475 et C478, Tab. 2 et Fig. 3) dont l'équivalent le plus proche est la lave du Côme : à titre de comparaison, le Tableau 2 donne des analyses de lave échantillonnée dans des coulées émergeant du Côme (C466, C467) et de lapilli scoriacés échantillonnés à mipente occidentale du volcan, près de la surface $(\mathrm{C} 445, \mathrm{C} 446)$ ou sous une coulée (C447, prélevé sous C466). L'ensemble des mesures, reportées sur un diagramme $\mathrm{CaO}$ vs $\mathrm{SiO}_{2}(\mathrm{Fig}$. 3), montre un bon regroupement de toutes ces compositions. Ceci supporte l'hypothèse que cette formation $\left(\mathrm{Ls}_{1}-\mathrm{Ls}_{2}\right.$ et $\left.\mathrm{Lr}_{3}\right)$ est issue du Puy de Côme, les produits de débourrage trouvés à sa base, en S16, correspondant à une phase d'ouverture du cratère.

La nature des lapilli squelettiques $\mathrm{Ls}_{0}$ conduit à rechercher leur origine dans un édifice trachybasaltique (C443). Le plus proche est le Filhu. Ce n'est pas le seul possible : la pente ouest du Puy Salomon (site S13), recèle des lapilli scoriacés rouges (donc proches de la source), immédiatement sous jacents aux produits de déferlantes du Kilian, de composition chimique très proche de celle de $\mathrm{Ls}_{0}$ (C386, Tab. 2).

\section{5 - Téphras sus-jacents à la formation du Pariou}

En plusieurs points, la formation $\mathrm{Lr}_{0}$ est couverte par un horizon brun, pluri-décimétrique, de consistance argileuse, contenant des petits fragments de socle et de laves diverses ainsi que des petites bombes trachytiques et des nodules de trachyte altéré (Tk, Fig. 2). De tels horizons sont fréquemment rencontrés dans les parties distales des nappes de déferlantes du Chopine, du Vasset ou du Kilian. C'est à ce dernier que peuvent probablement être rattachés ceux qui sont visibles, par endroits, dans le passage entre le Filhu et le Balmet (S5) et aux pieds ouest du Balmet (S6). La ligne de partage entre $\mathrm{Lr}_{0}$ et $\mathrm{Tk}$ n'est jamais très franche et les produits $\mathrm{Lr}_{0}$ sont généralement indurés et altérés à ce niveau.

Très ponctuellement, sur quelques mètres de longueur, dans le talus du chemin des Chaves entre le Filhu et le Balmet (S5, Fig. 2) l'horizon à trachytes Tk est lui-même recouvert par une formation décimétrique de lapilli à faciès de débourrage qui représentent donc l'une des dernières éruptions dans le centre de la Chaîne des Puys (« téphra des Chaves », $\mathrm{Lr}_{4}$, étude en cours). 
A la base septentrionale du puy de Dôme, le Nid de la Poule, est un cratère de $60 \mathrm{~m}$ de diamètre, couronné sur la moitié de son pourtour par une formation métrique de tufs compacts, gris-beiges, faiblement lités, attribuables à une éruption phréatomagmatique à magma basaltique (Boivin et al., 2004). Localement, au sud du cratère, ces tufs reposent sur la formation $\mathrm{Lr}_{0}$ du Pariou (S18, Tab. 2). Epaisse de plus de deux mètres, elle ne présente là que le sommet de la séquence, sans le lit basal de ponces. L'analyse élémentaire d'un lit décimétrique de lapilli scoriacés juvéniles, interstratifiés dans les produits de débourrage, s'inscrit parfaitement dans la série du Pariou, avec une composition de trachy-andésite (C523, Tab. 2 et Fig. 3).

\section{6-Téphrostratigraphie}

La pédogénèse superficielle du trachyte du Cliersou (paléosol, Fig. 2) suggère l'existence d'une période calme entre son éruption et celle qui a mis en place les produits remaniés $\operatorname{Lr}_{1}$. Pour comparaison, on peut se référer au sol observable au sommet du Puy de Dôme, sous les produits du Kilian (Raynal et al., 1998) : avec une épaisseur de 5 à $30 \mathrm{~cm}$, il représente environ 1500 ans (Cf. Boivin et al., 2004). On en déduit, par comparaison, que la période de repos qui a suivi l'éruption du Cliersou est au moins de quelques siècles, ce qui conduit à donner à ce dernier un âge minimum de 10000 ans environ.

Le sol superficiel du Cliersou est le seul sol qui ait été observé sous les téphras du Pariou dans le secteur étudié. Ceci suggère que l'éruption initiale du Pariou a succédé rapidement à une série d'événements volcaniques suffisamment rapprochés les uns des autres pour qu'aucun sol n'ait eu le temps de se développer.

La datation du puy de Côme reste problématique. Dans la carrière de Lantégy, des produits attribués au Côme sont recouverts par ceux du Chopine, âgé de 9700 ans environ (De Goër et al., 1999, Miallier et al., 2004). Guérin (1983) a obtenu par thermoluminescence (TL) les âges suivants : $11600 \pm 830(1 \sigma, \mathrm{Cp} 23)$ sur la coulée à Pontgibault, $15900 \pm 1500(\mathrm{Cp} 89)$ sur la coulée dans la forêt de Souis et $7610 \pm 1000$ (Cp58) sur un fragment de scorie rouge échantillonnée à $50 \mathrm{~cm}$ de profondeur sur le bord interne du cratère sommital (Guérin, com. pers.). Un âge TL de $12000 \pm 1500$ (LPC Cler.300) a été obtenu sur des lapilli attribués au Côme, échantillonnés sur le site de Vulcania (Saint-Ours-les-Roches) (Vernet et al., 2001). Enfin, l'observation de figures de colluvionnement cycliques marquant les produits du Côme dans la carrière de Lantégy a conduit De Goër et al. (1999) à privilégier une datation en climat pléniglaciaire, vers 15000 ans environ. Ainsi, compte tenu des différentes incertitudes, l'âge 
du Côme se trouverait dans la fourchette 12-15 ka environ. Toutefois, l'hypothèse d'une éruption sommitale tardive, émise initialement par Camus (1975), n’a pas été totalement abandonnée (Boivin et al., 2004) et elle est soutenue par la mesure TL à 7610 ans.

Or, la découverte récente de trachytes attribuables au Kilian dans le cratère terminal du Côme a indiqué un âge supérieur à celui du Kilian $(9,43 \pm 0,11 \mathrm{ka})$. Cette limite peut finalement être reportée à plus de 9500 ans, âge approximatif du Pariou dont les produits sont aussi présents sur le Côme. La mesure d'âge à 7610 ans (CP58) pourrait être compatible avec une éruption précédant de peu celle du Pariou si on élargit son incertitude à $2 \sigma$, soit \pm 2000 ans. Il faudrait dans cette hypothèse, et en conservant du crédit aux âges plus anciens, envisager que l'histoire du Côme est plus complexe que dans l'interprétation qui en est faite actuellement.

Le cratère du Nid de la Poule était considéré comme postérieur au puy de Dôme et, de peu, antérieur au Kilian (Camus, 1975 ; Boivin et al., 2004). La présence de trachytes attribuables au Kilian est en effet attestée sur ce cratère (Camus, 1975). Il est aujourd'hui possible de préciser qu'il est postérieur au Pariou dont il a localement recouvert les téphras, ce qui conduit à le situer dans une fourchette chronologique étroite, vers 9500-9400 ans environ.

\section{7 - Une faille affectant la formation du Pariou}

Au lieu dit La Plaine (S7), au sud du Puy Filhu, les téphras du Pariou, comprenant les lapilli à faciès de débourrage associant quelques ponces, et, au dessus, des lapilli de trachy-andésite (C430, Table 2), reposent soit sur des lapilli scoriacés noirs à faciès de saupoudrage, soit directement sur une coulée de lave d'origine et d'âge inconnus. A cet endroit, la coupe du terrain (orientée $100^{\circ} \mathrm{N}$ ), offre une structure remarquable : la séquence des téphras présente une figure d'affaissement vertical de $45 \mathrm{~cm}$ de déplacement et 1,6 m de largeur (Fig. 5). Cette structure a été dégagée jusqu'à 1,6 m sous la surface du sol, niveau où apparaît, de part et d'autre de la colonne de téphras affaissés, la lave massive, en place. On a pu vérifier, sur 1,5 $\mathrm{m}$ de longueur, que le bord $\mathrm{E}$ de cette structure est orienté $10^{\circ} \mathrm{N}$. On peut l'interpréter comme consécutive à l'ouverture d'une fissure dans la coulée, postérieurement à la mise en place de la formation du Pariou. Pour comparaison, à $2 \mathrm{~km}$ au nord de ce site, trois failles ouvertes de direction NNE-SSW affectent les coulées des volcans de Lantégy : deux sont situées sur le site de Vulcania et la troisième sur le volcan lui-même (De Goër et al., 1999). De Goër rattache ces failles à une fracture régionale majeure dite «faille de Saint-Sauves Aigueperse ». Il en déduit que cette fracture a rejoué depuis 30.000 ans environ, âge approximatif des deux volcans de Lantégy (Boivin et al., 2004, Guérin, 1983, et LPC Cler.311) «et peut-être 
beaucoup plus récemment ». Un segment, au moins, de cette faille aurait donc rejoué il y a moins de 9500 ans.

\section{8 -Conclusions}

Les nouvelles observations montrent l'existence d'une nappe occidentale, bien développée, de téphras liés à l'édification de l'anneau de tufs du Pariou et aux prémices du cône. La séquence, initiée par un lit de grosses ponces trachytiques de couleur claire, présente un empilement de lits de lapilli dont les caractéristiques témoignent d'une alternance de phases phréatomagmatiques ou magmatiques. La mise en place de ces produits s'est faite concurremment suivant un mode de retombées ou de déferlantes. Elle a été contrainte par le relief préexistant, de telle sorte que les téphras sont très inégalement répartis sur le terrain.

Cette formation résulte de la vidange d'un réservoir zoné par une succession d'explosions au cours desquelles une arrivée d'eau, plus ou moins contrariée suivant les phases, pouvait influer sur la dynamique éruptive. L'interaction entre des magmas différents dont témoignent les rubannements des ponces a pu contribuer plus modestement au déclenchement des explosions en créant un déséquilibre thermodynamique et chimique (Gourgaud et al., 1989).

Les transitions entre les différents magmas restent mal comprises; à cet égard une multiplication des analyses serait utile, dans la coupe S4 et dans les strates supérieures de l'anneau de tufs.

L'identification aisée de la formation en fait un bon marqueur téphrostratigraphique. A partir de ce repère, le schéma téphrochronologique du centre de la Chaîne des Puys a pu être précisé sur deux points : (1) La dernière éruption du Puy de Côme s'est produite il y a plus de c. 9500 ans. (2) Le Nid de la Poule s'est ouvert durant le bref intervalle - quelques siècles au maximum - qui a séparé l'éruption du Pariou de celle du Kilian, soit dans la fourchette 95009400 ans environ. En outre, un événement tectonique a engendré l'ouverture d'une faille dans le centre de la Chaîne des Puys, il y a moins de c. 9500 ans. 


\section{Références}

Boivin P., Besson J.C., Briot D., Camus G., de Goër A., Gourgaud A., Labazuy P., de Larouzière D., Livet M., Mergoil J., Miallier D., Morel J.M., Vernet G., Vincent P., 2004 Volcanologie de la Chaîne des Puys, carte et fascicule, $4^{\circ}$ édition, Parc Naturel des Volcans d'Auvergne (ed.), $179 \mathrm{p}$.

Camus G., 1975 - La Chaîne des Puys (Massif-Central Français). Etude structurale et volcanologique. Thèse de Doctorat. Annales de l'Université de Clermont-Ferrand, 56 (28), série Géologie et Minéralogie, $322 \mathrm{p}$.

Camus G., de Goër A., Kieffer G., Mergoil J. Vincent P.-M., 1982 -Volcanologie de la Chaîne des Puys, carte et fascicule, $2^{\circ}$ édition, Parc Naturel des Volcans d'Auvergne (ed.), $112 \mathrm{p}$

Choupin L. 2005 - Etude volcanologique de téphras liés à l'éruption du Pariou. Mémoire d'études et de recherches, LMV, Université Blaise Pascal, Clermont-Ferrand, 23 p.

El Arabi A., 1987 - Différentiation d'une série alcaline continentale. Exemple du puy de Pariou. Rapport de DEA, Université Blaise Pascal, Clermont-Ferrand, $50 \mathrm{p}$.

Fisher R.V. and Schmincke H.U., 1984 - Pyroclastic rocks, Springer Verlag (ed.), Berlin Heidelberg, $472 \mathrm{p}$.

Goër de Hervé A., Camus G., Lavina P., Michelin Y., Montel J.L., Tort M., 1999 - Lemptégy, volcan à ciel ouvert pour comprendre la Chaîne des Puys. S.a.r.l. Volcan de Lemptégy, Orcines (ed.), $56 \mathrm{p}$.

Gourgaud A., Fichaut M. et Joron J.-L., 1989 - Magmatology of Mt. Pelée (Martinique (F.W.I.). I : magma mixing and triggering of the 1902 and 1929 Pelean nuées ardentes, $J$. Volc., Geoth. Research, 38, 143-169. 
Guérin G., 1983 - La thermoluminescence des plagioclases, méthode de datation du volcanisme. Applications au domaine volcanique français : Chaîne des Puys, Mont Dore et Cézallier, Bas Vivarais. Thèse, Université Pierre et Marie Curie, Paris, 260 p.

Malterre D., 2005 - Etude volcanologique de téphras liés à l'éruption du Pariou. Mémoire d'études et de recherches, LMV, Université Blaise Pascal, Clermont-Ferrand, 23 p.

Miallier D., Michon L., Evin J., Pilleyre T., Sanzelle S. et Vernet G., 2004 - Volcans de la Chaîne des Puys (Massif Central, France) : point sur la chronologie Chopine-Pariou-VassetKilian. Comptes Rendus Acad. Sc., Géoscience, 336, 1345-1353.

Raynal J.P., Vernet G., Vivent D., 1998 - Des volcans et des hommes depuis le dernier interglaciaire en Auvergne (Massif Central, France). In : Centro Universitario Europeo per il Beni Culturali, Ravello. Edipuglia Bari, (ed.). Il Sistema Uomo-Ambiante Tra Passato E Presente, sous la direction de C. Albore Livadie et F. Ortolani, 199-220.

Vernet G., Raynal J.P., Kieffer G., Camus G., Guadelli J.L., Vivent D., Miallier D., Sanzelle S., Pilleyre T., Faïn J., Montret M., 2001 - L'éruption du Puy Chopine (Puy de Dôme, France) : impacts proximaux et distaux et implications éco-archéologiques. In : CDRAD, 43150 Goudet (ed.), Tephras, Actes du symposium INQUA-UISPP, Le Puy en Velay, 24-29 Août $1998,2001,227-234$

Vernet G. et Raynal J.P., 2000 - Un cadre téphrostratigraphique réactualisé pour la préhistoire tardiglaciaire et holocène de Limagne (Massif-Central, France). Comptes Rendus Acad. Sc., Sciences de la Terre et des planètes, Paris, 330, 399-405.

Wohletz K. and Brown W., 1995 - Particulate size distribution and sequential fragmentation/transport theory, Rapport interne LA-UR 95-0371, Los Alamos National Laboratory, $9 \mathrm{p}$.

Wohletz K. and Heiken G., 1985 - Vocanic Ash, University of California Press (ed.), 246 p. 


\begin{tabular}{|l|l|l|l|}
\hline Référence & \multicolumn{2}{|c|}{ Site } & \multicolumn{2}{c|}{ Coordonnées UTM } \\
\hline S1 & Cliersou & 496850 & 5071170 \\
\hline S2 & Petit Suchet & 496977 & 5070657 \\
\hline S3 & Col Côme-Balmet & 495276 & 5070905 \\
\hline S4 & Carrière Filhu-Balmet & 495253 & 5070240 \\
\hline S5 & Chemin des Chaves & 495012 & 5070200 \\
\hline S6 & Chemin du bac de Ceyssat & 494750 & 5070585 \\
\hline S7 & La Plaine & 495510 & 5069291 \\
\hline S8 & Sentier du Côme & 495145 & 5071449 \\
\hline S9 & Carrière NW Côme & 494804 & 5071364 \\
\hline S10 & Col Côme Balmet (coulée) & 495272 & 5070918 \\
\hline S11 & Sentier du Côme & 495146 & 5071074 \\
\hline S12 & Sentier du Côme & 495107 & 5071202 \\
\hline S13 & Cratère de Salomon & 495615 & 5066937 \\
\hline S14 & Base du Petit Sault & 495460 & 5068694 \\
\hline S15 & Captage Montmeyre & 494961 & 5069896 \\
\hline S16 & Col Filhu-Balmet & 495457 & 5070277 \\
\hline S18 & Nid de la Poule & 497326 & 5069460 \\
\hline S19 & La Pépinière de Royat & 502094 & 5067278 \\
\hline
\end{tabular}

Tab. 1 . Localisation des coupes étudiées. 


\begin{tabular}{|c|c|c|c|c|c|c|c|c|c|c|c|c|c|c|c|}
\hline réf. & site & lit & nature & SiO2 & Al2O3 & Fe2O3 & MnO & MgO & CaO & Na2O & K2O & TiO2 & P2O5 & PF & total \\
\hline C 344-5a & S19 & & Ls & 47,95 & 19,52 & 9,9 & 0,22 & 2,62 & 6,43 & 3,33 & 1,58 & 1,76 & 0,64 & 6,04 & 99,99 \\
\hline C 344-5b & S19 & & Ls & 50,82 & 18,47 & 9,87 & 0,23 & 3,02 & 6,99 & 4,14 & 2,18 & 1,73 & 0,68 & 1,85 & 99,98 \\
\hline C 344-1 & S19 & & Ls & 47,74 & 17,71 & 11,7 & 0,21 & 3,86 & 7,06 & 3,68 & 2,02 & 2,12 & 0,71 & 3,09 & 99,90 \\
\hline C 430 & S7 & Lr0 & Ls & 50,18 & 17,88 & 10,19 & 0,22 & 3,18 & 6,84 & 4,46 & 2,42 & 1,78 & 1,03 & 0,94 & 99,13 \\
\hline C 523 & S18 & Lr0 & Ls & 51,27 & 17,47 & 10,11 & 0,22 & 3,18 & 6,83 & 4,65 & 2,57 & 1,77 & 1,03 & 0,44 & 99,53 \\
\hline C 480 & S4 & Lr0 & Ls & 51,75 & 17,66 & 10,21 & 0,22 & 3,00 & 6,70 & 4,66 & 2,67 & 1,73 & 1,00 & $-0,14$ & 99,44 \\
\hline C 445-a & S12 & & Ls & 49,83 & 17,14 & 10,81 & 0,22 & 4,02 & 7,62 & 4,19 & 2,38 & 2,02 & 0,83 & 0,19 & 99,26 \\
\hline C 446 & S11 & & Ls & 50,09 & 17,62 & 10,42 & 0,22 & 3,87 & 7,43 & 3,97 & 2,15 & 2,02 & 0,77 & 1,47 & 100,02 \\
\hline C 447 & S10 & & Ls & 47,10 & 16,83 & 11,49 & 0,22 & 4,68 & 8,16 & 3,55 & 1,90 & 2,21 & 0,83 & 2,55 & 99,51 \\
\hline C 464 & S6 & Ls2 & Ls & 48,58 & 17,70 & 11,12 & 0,22 & 4,22 & 8,15 & 3,69 & 1,81 & 2,12 & 0,79 & 2,22 & 100,61 \\
\hline C 466 & S10 & & Lm & 49,19 & 17,04 & 10,90 & 0,21 & 4,62 & 8,44 & 3,96 & 2,18 & 2,21 & 0,82 & 0,17 & 99,73 \\
\hline C 467 & S9 & & Lm & 50,16 & 16,94 & 10,76 & 0,21 & 4,53 & 8,27 & 4,19 & 2,29 & 2,21 & 0,85 & 0,11 & 100,52 \\
\hline C 475 & S4 & Ls2 & Ls & 50,64 & 17,23 & 10,87 & 0,22 & 4,21 & 8,41 & 4,14 & 2,14 & 2,03 & 0,75 & $-0,07$ & 100,56 \\
\hline C 478 & S4 & Ls2 & Ls & 51,63 & 17,67 & 10,27 & 0,22 & 3,73 & 7,66 & 4,26 & 2,39 & 1,94 & 0,82 & 0,13 & 100,71 \\
\hline C 482 & S3 & Ls1 & Ls & 47,21 & 17,71 & 11,47 & 0,22 & 4,20 & 7,71 & 3,48 & 1,80 & 2,24 & 0,83 & 3,20 & 100,05 \\
\hline C 443 & S3 & Ls0 & Ls & 44,85 & 17,32 & 12,45 & 0,22 & 4,20 & 7,39 & 3,35 & 1,76 & 2,44 & 0,91 & 3,99 & 98,89 \\
\hline C 386 & S13 & & Ls & 45,92 & 18 & 12,56 & 0,22 & 4,13 & 7,24 & 3,59 & 1,82 & 2,4 & 0,78 & 2,72 & 99,38 \\
\hline
\end{tabular}

Tab. 2. Analyses élémentaires, effectuées au Centre de Recherches Pétrographiques et Géochimiques (CRPG), Vandoeuvre-lès-Nancy. Nature de l'échantillon : Ls lapilli scoriacés ; Lm lave massive (d'une coulée). Les échantillons sont regroupés conformément aux apparentements supposés ou avérés (voir le texte). 


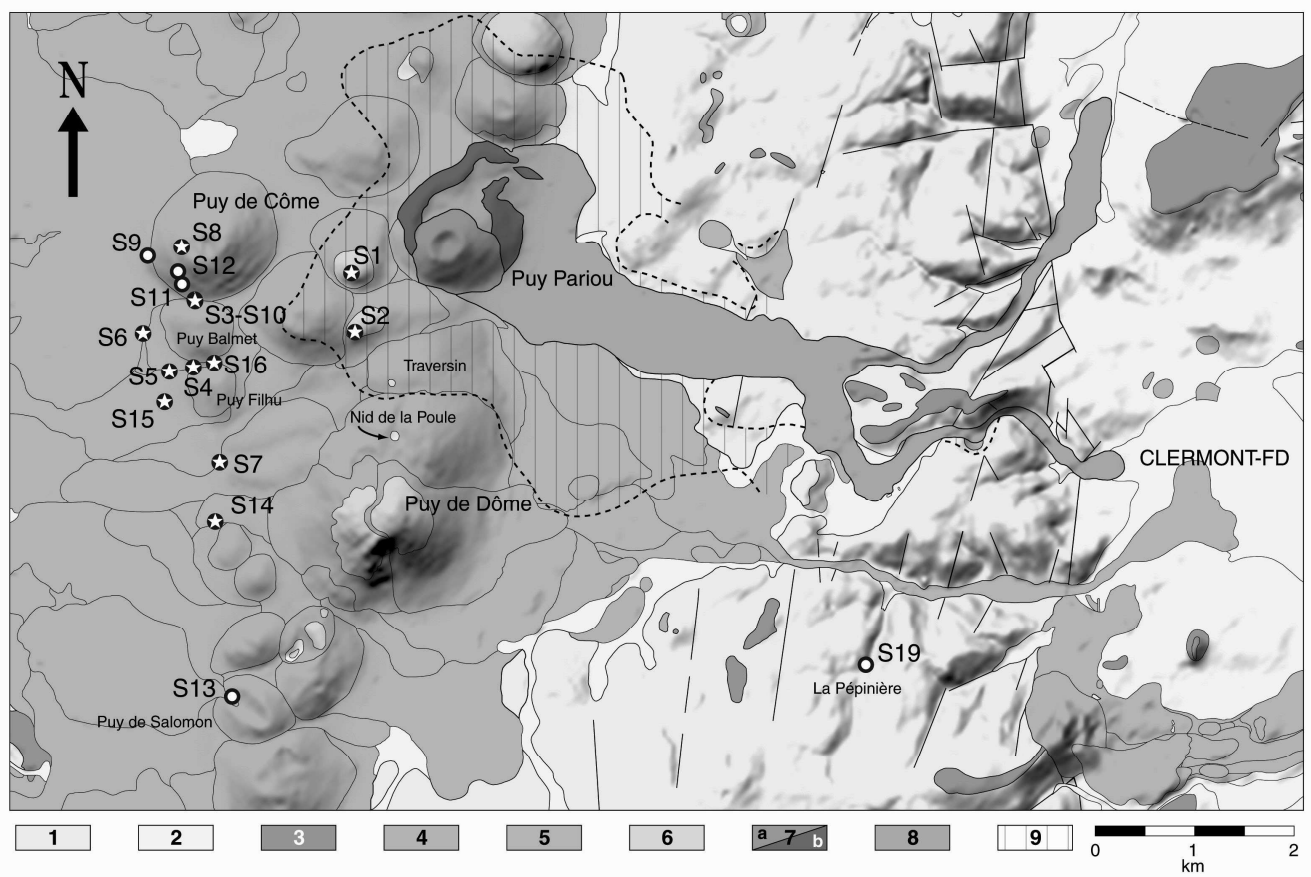

Figure 1. Carte avec localisation des sites d'observation (S).

\section{Légende}

1 Socle

2 Sédiments

3 Volcanisme antérieur à la Chaîne des Puys

Formations indifférenciées de la Chaîne des Puys

4 Pyroclastites

5 Coulées de laves

6 Dômes

Formations du système volcanique du Puy Pariou

7 Pyroclastites : a) cône strombolien; b) anneau de tuf initial.

8 Coulées de trachy-andésite

9 Limite de la zone d'extension principale des tufs de l'anneau antérieurement connue [1]

Étoiles : nouveaux sites décrits ici où s'observent les dépôts du Puy Pariou

Ronds : autres lieux cités 


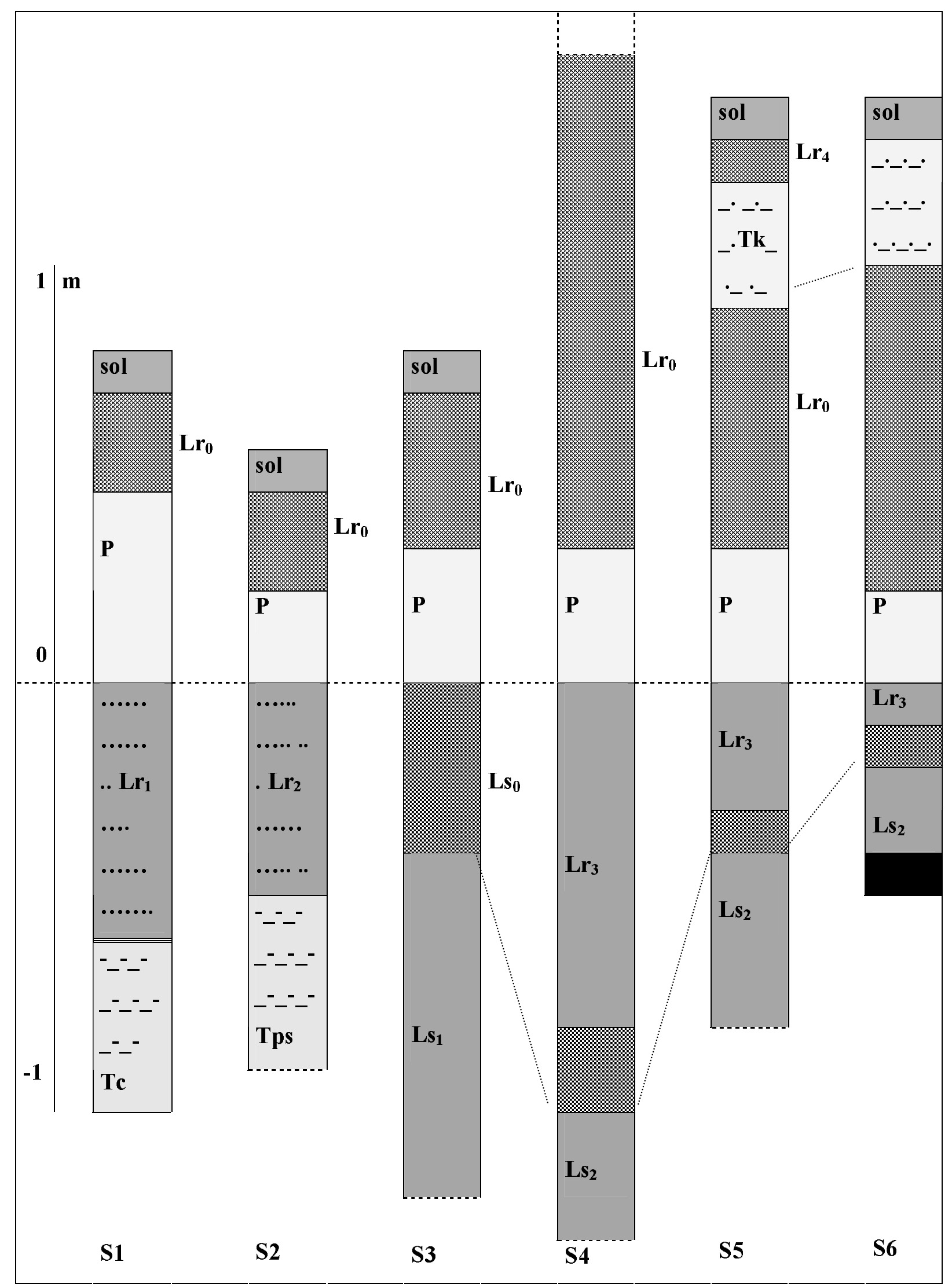




\section{Fig. 2.}

Téphrostratigraphie des sites S1 à S6 (voir Tab. 1). La formation décrite dans le texte $(\S 2)$ commence par les ponces, $\mathrm{P}$, et se poursuit par une succession de lits de lapilli à faciès de débourrage, plus ou moins riches en lave juvénile: $\operatorname{Lr}_{0} . \operatorname{Lr}_{1}$ et $\operatorname{Lr}_{2}$ : lapilli à faciès de débourrage sur le Cliersou et le Petit Suchet; Tc et Tps : trachytes massifs du Cliersou, pédogénisé en surface (traits), et du Petit Suchet. $\mathrm{Ls}_{0}$ : lapilli squelettiques à faciès de saupoudrage et composition de trachy-basalte. $\mathrm{Ls}_{1}, \mathrm{Ls}_{2}$ : lapilli scoriacés à composition de trachyandésite. $\mathrm{Lr}_{3}$ lapilli à faciès de débourrage. $\mathrm{Lr}_{4}$ : téphra récent du chemin des Chaves (lapilli à faciès de débourrage). Tk : cendres trachytiques altérées contenant des nodules de trachyte plus ou moins altéré, type Kilian. En noir, site S6 : surface de coulée de lave, base non observée.

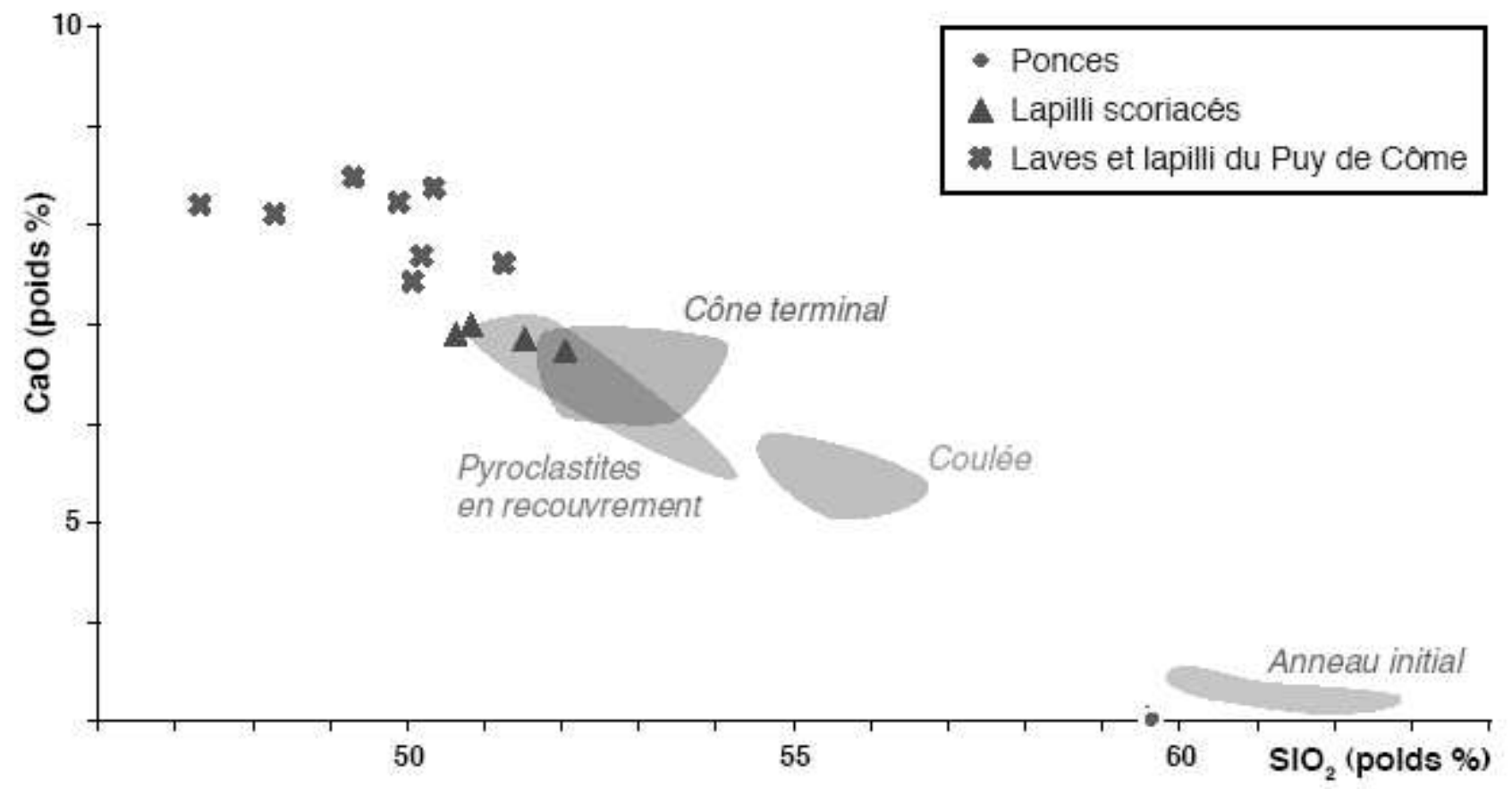

Figure 3. Diagramme $\mathrm{CaO}$ vs $\mathrm{SiO}_{2}$. Les données de la littérature pour le Pariou (nuages) sont comparées aux analyses récentes (Tab. 2) : produits associés au puy de Côme, lapilli de la formation du Pariou et ponces. 


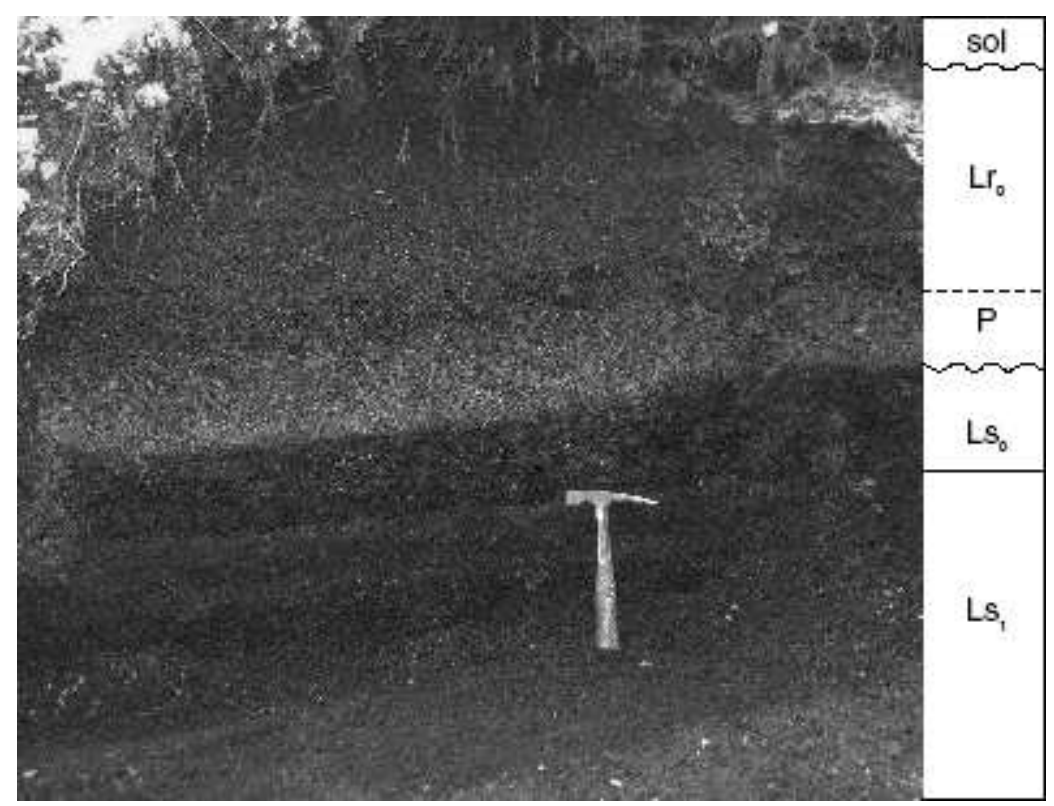

Figure 4. Coupe de talus au col entre le puy de Côme et le Puy Balmet (S3, Fig. 2. Le lit de ponces est le plus clair. L'association des ponces et du premier lit de lapilli noirs, au dessus, forme un repère très caractéristique sur le terrain. 

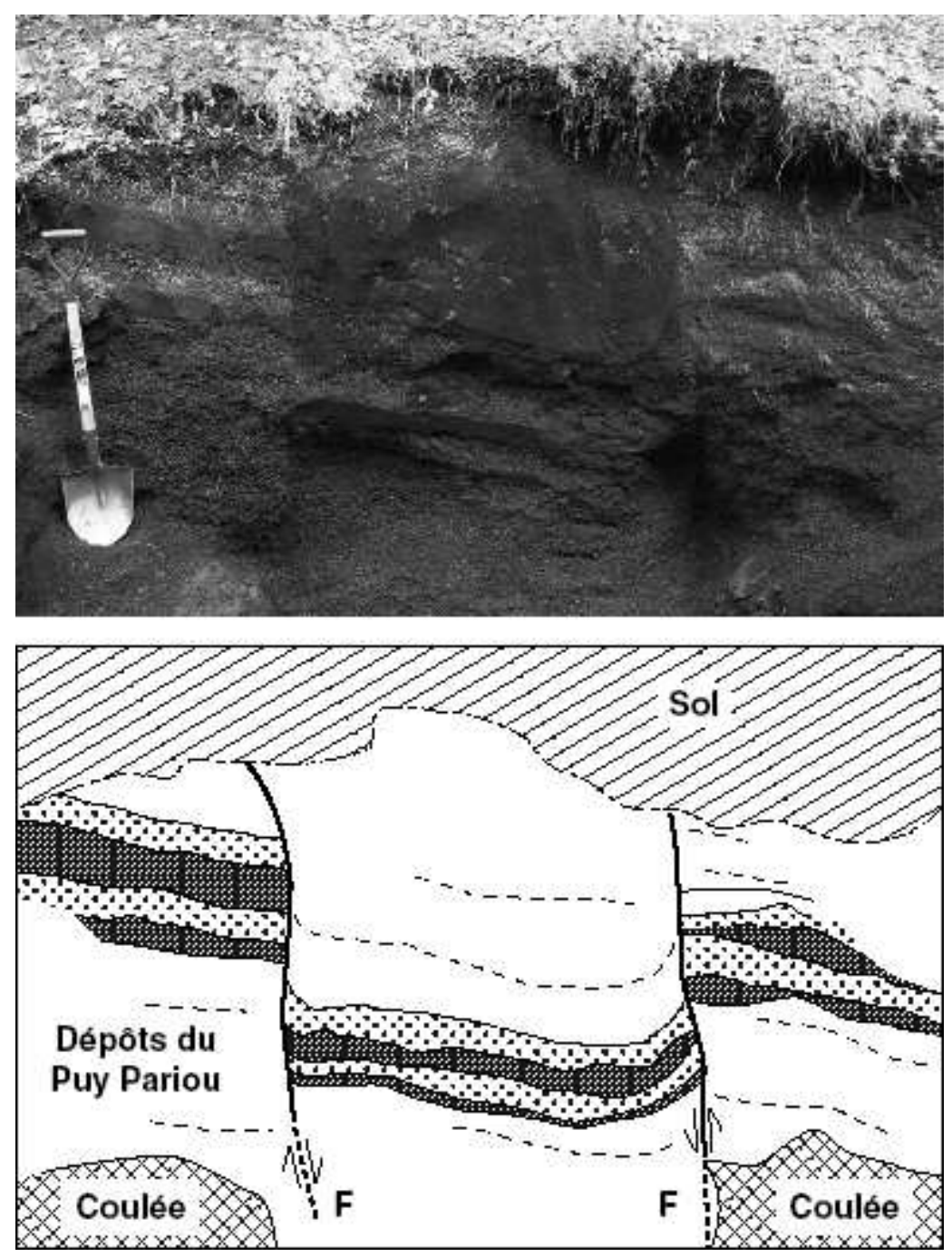

Figure 5. Figure d'affaissement vertical dans la formation du Pariou. Lieu-dit La Plaine, site S7. Largeur : $1,60 \mathrm{~m}$ 